between such functions as $u$ and $\tilde{u}$ (the representative function agreeing with $u$ on $\mu_{\delta}$ and approaching $u$ in the norm) the proof runs as follows:

From (23), there is, for any $\epsilon>0$, a $\delta_{0}$ such that, for $\delta<\delta_{0}$, $\left\|w-S_{\delta}^{-1} u\right\|<\epsilon$ where we write $A u=w, S w=u$. On multiplying $w^{\prime}=\left(w-\tilde{S}_{\delta}^{-1} u\right)$ by $\tilde{S}_{\delta}$ one has, from (24) $\left\|\tilde{S}_{\delta} w-u\right\|=\left\|S_{\delta} w^{\prime}\right\| \leqq M^{\prime}\left\|w^{\prime}\right\|$ $<M^{\prime} \epsilon$ which gives (25) and the scheme is convergent.

As in Part I, the converse follows from the Principle of Uniform Boundedness.

\title{
REFERENCE
}

1. P. Lax and R. Richtmeyer, Survey of the stability of linear finite difference equations, Comm. Pure Appl. Math. vol. 9 (1956) pp. 267-293.

University of CALIFornia, Livermore

\section{DUALITY IN HOMOGENEOUS PROGRAMMING ${ }^{1}$}

\section{E. EISENBERG}

The problem of maximizing a concave function subject to linear constraints does not have a dual, as is the case in linear programming, in which primal optimizing variables do not appear. As a special case of our principal result it will follow that such a dual does indeed exist whenever the objective function is also homogeneous.

In the linear case we are given an $m \times n$ matrix $A$ and vectors $a \in R^{n}, b \in R^{m} .^{2}$ The feasibility sets $X$ and $Y$ are defined by: $X=R_{+}^{m} \cap\{x \mid x A \leqq a\}, Y=R_{+}^{n} \cap\{y \mid A y \geqq b\}$. Since $x A \leqq a$ if and only if $x A y \leqq a y$ for all $y \in R_{+}^{n}$ (and similarly for $A y \geqq b$ ), we may write:

$$
\begin{aligned}
& X=R_{+}^{m} \cap\left\{x \mid x A y \leqq \psi(y) \quad \text { all } y \in R_{+}^{n}\right\} \\
& Y=R_{+}^{n} \cap\left\{y \mid x A y \geqq \phi(x) \quad \text { all } x \in R_{+}^{m}\right\}
\end{aligned}
$$

Received by the editors April 26, 1960; and in revised form, July 27, 1960 and September 12, 1960.

${ }^{1}$ Work on this paper was supported, in part, by the Logistics Branch of the Office of Naval Research under Contract NONR 562(15) at Brown University.

${ }^{2} R^{m}$ denotes the set of all real $m$-tuples. If $u, v \in R^{m}$ then $u \leqq v$ means that the inequality holds for each component. In particular, $R_{+}^{m}=R^{m} \bigcap\{x \mid x \geqq 0\}$. If $M$ is a $p \times q$ matrix and $N$ is a $q \times t$ matrix then $M N$ represents the usual matrix product. To simplify notation, the same symbol is used for both a column vector and its transpose; the meaning will, in any case, be clear from the context. 
where $\psi(y)=a y$ and $\phi(x)=b x$.

A fundamental theorem of linear programming (see, e.g., $[3 ; 2]$ ) states that if $X$ and $Y$ are both nonempty then

$$
\max _{x \in X} \phi(x), \min _{y \in Y} \psi(y) \text { exist and are equal. }
$$

We propose to demonstrate that (2) holds for another class of triples $(A, \phi, \psi)$.

Assumption $A_{1}$. Let $\phi: R_{+}^{m} \rightarrow R, \psi: R_{+}^{n} \rightarrow R$ be positively homogeneous, ${ }^{3}$ continuous, concave and convex respectively.

Let us first show that $A_{1}$ does not guarantee that (2) holds when $X$ and $Y$ are nonempty. If $m=2, n=1$ and

$$
A=\left[\begin{array}{l}
0 \\
1
\end{array}\right] \text {, }
$$

$\phi(x)=\phi(\xi, \eta)=\xi \eta /(\xi+\eta)(\phi(0)=0), \psi(y)=y$ then $A_{1}$ is satisfied and $X=R_{+}^{2} \cap\{(\xi, \eta) \mid \eta \leqq 1\}, \quad Y=R_{+} \cap\{y \mid y \geqq 1\}$ are nonempty. Thus $\min _{y \in Y} \psi(y)=1$, but if $\eta \leqq 1$ then $\phi(\xi, \eta)<1$, although $\sup _{x \in X} \phi(x)=1$, hence $\max _{x \in X} \phi(x)$ does not exist.

The situation just illustrated cannot occur if the following holds:

Assumption $\mathrm{A}_{2}$.

(i) If $x \in R_{+}^{m}, x A \leqq 0, \phi(x) \geqq 0$ then $x=0$.

(ii) If $x \in R_{+}^{n}, A y \geqq 0, \psi(y) \leqq 0$ then $y=0$.

One sees immediately that (i) is violated in the preceding example, for let $x=(1,0)$ then $x A=0$ and $\phi(x)=0$.

Before proving our main result, that if $A_{1}$ and $A_{2}$ hold then so does (2), we require the following lemma which specializes to homogeneous functions the well-known fact that a concave function is the infimum of its supports. The proof is presented here for the sake of completeness.

Lemma. Let $\phi$ be as in assumption $\mathrm{A}_{1}$, consider

$$
T=R^{m} \cap\left\{t \mid t x \geqq \phi(x) \text { all } x \in R_{+}^{m}\right\},
$$

then $T$ is nonempty, and $\phi(x)=\inf _{t \in T} t x$, for all $x \in R_{+}^{m}$.

Proof. Let $C=\left\{(x, \lambda) \mid x \in R_{+}^{m}, \lambda \leqq \phi(x)\right\}$ then $C$ is a closed convex cone. Now if $x_{0} \in R_{+}^{m}, \epsilon>0$, then $\left(x_{0}, \epsilon+\phi\left(x_{0}\right)\right) \notin C$, whence (see [2, Theorem 1]) there exist $t \in R^{m}$ and $\alpha \in R$ such that $t x_{0}-\alpha\left[\epsilon+\phi\left(x_{0}\right)\right]$ $<0 \leqq t x-\alpha \lambda$ all $(x, \lambda) \in C$.

${ }^{3}$ A function $f: C \rightarrow R q$, where $C \subset R^{p}$ is a cone, is positively homogeneous providing $f(\lambda x)=\lambda f(x)$ for all $x \in C$ and $\lambda \in R_{+}$. 
It then follows that $\alpha>0$, so that (dividing by $\alpha$ ) we may assume $\alpha=1$, but then $t \in T$. Reiterating, if $x_{0} \in R_{+}^{m}, \epsilon>0$ then $\exists t \in T$ such that:

$$
t x_{0}-\epsilon \leqq \phi\left(x_{0}\right) \leqq t x_{0}
$$

giving the desired result. We are now able to prove:

TheOREM 1. If assumptions $\mathrm{A}_{1}$ and $\mathrm{A}_{2}$ hold then (2) holds.

ProOF. Let

$$
\begin{array}{ll}
S=R^{n} \cap\{s \mid s y \leqq \psi(y) & \text { all } \left.y \in R_{+}^{n}\right\}, \\
T=R^{m} \cap\{t \mid t x \geqq \phi(x) & \text { all } \left.x \in R_{+}^{m}\right\} .
\end{array}
$$

Then $S$ and $T$ are convex sets; now consider the system of inequalities:

$$
\begin{gathered}
x \in R_{+}^{m}, y \in R_{+}^{n}, s \in S, t \in T, \\
s-x A>0, \\
-t+A y>0, \\
\phi(x)-\psi(y)>0 .
\end{gathered}
$$

If (4) has a solution $x, y, s, t$ then

$$
\psi(y)<\phi(x) \leqq t x \leqq x A y \leqq s y \leqq \psi(y)
$$

which is a contradiction. Thus (see [1, Theorem 1]) there exist $x_{0} \in R_{+}^{m}, y_{0} \in R_{+}^{n}, \lambda \in R_{+}$, not all zero and such that $(s-x A) y_{0}$ $+x_{0}(A y-t)+\lambda[\phi(x)-\psi(y)] \leqq 0$ for all $x \in R_{+}^{m}, y \in R_{+}^{n}, s \in S, t \in T$. From the homogeneity and continuity of $\phi$ and $\psi$ it then follows that:

$$
\begin{array}{rr}
x A y_{0} \geqq \lambda \phi(x) & \text { all } x \in R_{+}^{m}, \\
x_{0} A y \leqq \lambda \psi(y) & \text { all } y \in R_{+}^{n}, \\
s y_{0} \leqq t x_{0} & \text { all } s \in S, t \in T .
\end{array}
$$

The last condition together with our lemma imply:

$$
\psi\left(y_{0}\right) \leqq \phi\left(x_{0}\right) .
$$

Now if $\lambda=0$ then either $x_{0} \neq 0$ or $y_{0} \neq 0$ and $A y_{0} \geqq 0, x_{0} A \leqq 0$. Suppose $x_{0} \neq 0$, then by $A_{2}$ (i) we have $\phi\left(x_{0}\right)<0$, whence $\psi\left(y_{0}\right)<0$ and $y_{0} \neq 0$, contradicting $A_{2}$ (ii). Thus $\lambda>0$ and, dividing all inequalities by $\lambda$, we may assume $\lambda=1$. This tells us that $x_{0} \in X, y_{0} \in Y$ and $\phi\left(x_{0}\right) \leqq x_{0} A y_{0} \leqq \psi\left(y_{0}\right) \leqq \phi\left(x_{0}\right)$. So that if $x \in X, y \in Y$ then 


$$
\begin{aligned}
& \phi(x) \leqq x A y_{0} \leqq \psi\left(y_{0}\right)=\phi\left(x_{0}\right), \\
& \psi(y) \geqq x_{0} A y \geqq \phi\left(x_{0}\right)=\psi\left(y_{0}\right)
\end{aligned}
$$

proving the theorem.

In case $\phi$ and $\psi$ are linear-homogeneous then it is true that $\max _{x \in X} \phi(x)$ exists if and only if $\min _{y \in Y} \psi(y)$ exists, in which case they are equal. As above, this statement is not true under assumption $A_{1}$; however, we show:

Theorem 2. (I) If $\mathrm{A}_{1}$ and $\mathrm{A}_{2}$ (ii) hold and $\max _{x \in X} \phi(x)$ exists then $\min _{y \in Y} \psi(y)$ exists and the two are equal.

(II) If $\mathrm{A}_{1}$ and $\mathrm{A}_{2}$ (i) hold and $\min _{y \in Y} \psi(y)$ exists then $\max _{x \in X} \phi(x)$ exists and the two are equal.

We prove (I), the proof of (II) is similar. Suppose that $x_{0} \in X$ and $\phi\left(x_{0}\right)=\max _{x \in X} \phi(x)$ then the system:

$$
\begin{gathered}
x \in R_{+}^{m}, s \in S \\
s-x A>0, \\
\phi(x)-\phi\left(x_{0}\right)>0,
\end{gathered}
$$

has no solution. Thus (see $\left[1\right.$, Theorem 1]) there exist $y_{0} \in R_{+}^{n}, \lambda \in R_{+}$, not both zero and such that

$$
s y_{0}-x A y_{0}+\lambda\left[\phi(x)-\phi\left(x_{0}\right)\right] \leqq 0 \quad \text { for all } x \in R_{+}^{m}, s \in S .
$$

From the homogeneity of $\phi$ and our lemma it then follows that

$$
\begin{array}{ll}
x A y_{0} \geqq \lambda \phi(x) & \text { for all } x \in R_{+}^{m}, \\
\psi\left(y_{0}\right) \leqq \lambda \phi\left(x_{0}\right) . &
\end{array}
$$

Now if $\lambda=0$ then $y_{0} \neq 0$ and $A y_{0} \geqq 0, \psi\left(y_{0}\right) \leqq 0$ contradicting $\mathrm{A}_{2}$ (ii). It may then be assumed that $\lambda>0$ and, in fact, that $\lambda=1$ (replacing $y_{0}$ by $\lambda y_{0}$ ). Thus, from (7), $y_{0} \in Y$ and for any $y \in Y$ we have:

$$
\psi\left(y_{0}\right) \leqq \phi\left(x_{0}\right) \leqq x_{0} A y \leqq \psi(y),
$$

i.e.,

$$
\psi\left(y_{0}\right)=\min _{y \in Y} \psi(y)=\phi\left(x_{0}\right) .
$$

It should be remarked that if $A_{1}$ holds then (i) and (ii) of assumption $A_{2}$ are equivalent to (i)' and (ii)' respectively of:

Assumption $\mathrm{A}_{2}^{\prime}$.

(i) $)^{\prime} \exists y_{0} \in R_{+}^{n} \ni x A y_{0}>\phi(x)$ all $x \in R_{+}^{m}, x \neq 0$.

(ii) $\exists x_{0} \in R_{+}^{m} \ni x_{0} A y<\psi(y)$ all $y \in R_{+}^{n}, y \neq 0_{s}$. 
These in turn are equivalent to the familiar conditions that $X, Y$ have nonempty interiors. To see, for instance, that (i) and (i)' are equivalent it suffices to show that (i) implies (i)' since the implication in the other direction is trivial. Assuming (i)' false, the system

$$
\begin{gathered}
y \in R_{+}^{n}, t \in T, \\
A y-t>0
\end{gathered}
$$

has no solution, whence (see [1, Theorem 1]) there is an $x \in R_{+}^{m}, x \neq 0$, and such that $x A y \leqq t x$ for all $y \in R_{+}^{n}$ and $t \in T$. Thus $x A \leqq 0$ and (using our lemma) $\phi(x) \geqq 0$, contradicting (i). To return to our remark about maximizing a concave homogeneous and continuous function $\phi: R_{+}^{m} \rightarrow R$, subject to the inequalities $x \geqq 0$ and $x A \leqq a$, the dual is then: minimize $a y$ subject to $y \in Y$. Conditions (i) and (ii)' become:

$$
\begin{aligned}
& x \in R_{+}^{m}, x \neq 0, x A \leqq 0, \phi(x) \geqq 0 \text { has no solution; and } \\
& x \in R_{+}^{m}, x A<a \text { has a solution; respectively. }
\end{aligned}
$$

Also, since $y \in Y$ providing $y \geqq 0$ and $A y \geqq t$ for some support $t$ of $\phi$, we may characterize $Y$ by means of the gradient of $\phi$.

\section{BIBLIOGRAPHY}

1. Ky Fan, I. Glicksberg, and A. J. Hoffman, Systems of inequalities involving convex functions, Proc. Amer. Math. Soc. vol. 8 (1957) pp. 617-622.

2. W. Fenchel, Convex cones, sets and functions. From notes by D. W. Blacket of Lectures at Princeton University, Spring 1953.

3. D. Gale, H. W. Kuhn, and A. W. Tucker, Linear programming and the theory of games. Activity Analysis of Production and Allocation. Cowles Commission Monograph 13, New York, 1951, pp. 317-329.

\section{BROWN UNIVERSITY AND}

Hughes RESEARCH LABORATORIES 